\title{
Decomposition and biodegradability enhancement of textile wastewater using a combination of electron beam irradiation and activated sludge process
}

\begin{abstract}
The research conducted a study on decomposition and biodegradability enhancement of textile wastewater using a combination of electron beam irradiation and activated sludge process. The purposes of this research are to remove pollutant through decomposition and to enhance the biodegradability of textile wastewater. The wastewater is treated using electron beam irradiation as a pre-treatment before undergo an activated sludge process. As a result, for non-irradiated wastewater, the COD removal was achieved to be between $70 \%$ and $79 \%$ after activated sludge process. The improvement of COD removal efficiency increased to $94 \%$ after irradiation of treated effluent at the dose of $50 \mathrm{kGy}$. Meanwhile, the $\mathrm{BOD}(5)$ removal efficiencies of nonirradiated and irradiated textile wastewater were reported to be between 80 and $87 \%$, and 82 and $99.2 \%$, respectively. The maximum BOD(5) removal efficiency was achieved at day 1 (HRT 5 days) of the process of an irradiated textile wastewater which is $99.2 \%$. The biodegradability ratio of non-irradiated wastewater was reported to be between 0.34 and 0.61 , while the value of biodegradability ratio of an irradiated wastewater increased to be between 0.87 and 0.96 . The biodegradability enhancement of textile wastewater is increased with increasing the doses. Therefore, an electron beam radiation holds a greatest application of removing pollutants and also on enhancing the biodegradability of textile wastewater.
\end{abstract}

Keyword: Activated sludge process; Biodegradability; Decomposition; Electron beam radiation 